\title{
Estimates of Sodium and Potassium intake and their molar ratio in Irish children: Preliminary findings from NCFSII
}

\author{
$\underline{\text { Eoin Morrissey }}^{1}$, Laura Kehoe ${ }^{2}$, Aoibhin Moore Heslin ${ }^{3}$, Maria Buffini $^{3}$, Breige McNulty ${ }^{3}$, \\ Anne Nugent ${ }^{4}$, John Kearney ${ }^{5}$, Albert Flynn ${ }^{2}$ and Janette Walton \\ ${ }^{1}$ Cork Institute of Technology, Cork, Ireland, \\ ${ }^{2}$ University College Cork, Cork, Ireland, \\ ${ }^{3}$ University College Dublin, Dublin, Ireland, \\ ${ }^{4}$ Queens University Belfast, Belfast, United Kingdom and \\ ${ }^{5}$ Technological University Dublin, Dublin, Ireland
}

\begin{abstract}
The Food Safety Authority of Ireland (FSAI) have set target maximum daily salt intakes for children (4-6y: 3 g, 7-10y: 5 g, 11-14y: $6 \mathrm{~g}$ ) while the European Food Safety Authority (EFSA) have set Adequate Intakes (AI) for potassium of 1100mg/d, 1800mg/d and $2700 \mathrm{mg} / \mathrm{d}$ for children of the same respective age groups. An individual's sodium to potassium (Na:K) intake ratio is an important predictor of hypertension and the World Health Organization (WHO) recommend a Na:K intake ratio of $\leq 1.0 \mathrm{mmol} / \mathrm{mmol}$ for both adults and children. Although the morbidities associated with hypertension may not be seen until adulthood, blood pressure in childhood has a significant association with blood pressure in adulthood. Therefore, estimation of Na:K intake ratios (best measured by urinary excretion) in children may predict their susceptibility to hypertension related diseases in later life. The aim of this study was to estimate sodium and potassium intake and mean molar $\mathrm{Na}: \mathrm{K}$ intake ratio of Irish children and to assess compliance with dietary guidance.

Morning spot urine samples were collected for 572 children aged 5-12 years ( $95 \%$ of total sample) as part of the nationally representative Irish National Children's Food Survey II (2017-2018) (NCFSII; www.iuna.net). Samples were transported, processed and stored using best practice procedures. Urinary excretion of sodium and potassium were measured using a Randox RX Daytona and were corrected for gender and age-specific 24-hour urine volume estimations based on 24-hour urine volume estimates from Australian children. SPSS Version 25 was used for all analyses.

Mean 24-hour urinary sodium excretion was $2018 \mathrm{mg} / \mathrm{d}$, equivalent to an average salt excretion of $5.0 \mathrm{~g} / \mathrm{d}$ exceeding the FSAI maximum target intake for all age groups except 11-12 year olds. Mean 24-hour urinary potassium excretion was $1411 \mathrm{mg} / \mathrm{d}$ with mean intakes below the AI from EFSA for all age groups with the exception of 5-6 year olds. The mean molar Na:K ratio of Irish children was 2.8 for boys and 3.4 for girls. Only $5 \%$ of Irish children met the WHO recommendation for a Na:K ratio of $\leq 1.0 \mathrm{mmol} / \mathrm{mmol}$.

High intakes of sodium and low intakes of potassium reported in this study result in a low compliance with the WHO recommendation of a Na:K ratio $\leq 1.0 \mathrm{mmol} / \mathrm{mmol}$. This may lead to a higher risk of hypertension and related morbidities in later life. Based on these findings, dietary interventions to combat hypertension related diseases (such as lowering sodium and increasing potassium intakes) should be implemented from childhood.
\end{abstract}

\section{Conflict of Interest}

There is no conflict of interest 